\title{
Cockayne syndrome type A: novel mutations in eight typical patients
}

\author{
Debora R. Bertola $\cdot$ Henian Cao $\cdot$ Lilian M. J. Albano • \\ Daniela P. Oliveira $\cdot$ Fernando Kok · \\ Maria Joaquina Marques-Dias · Chong A. Kim • \\ Robert A. Hegele
}

Received: 3 April 2006/ Accepted: 7 May 2006/Published online: 25 July 2006

(C) The Japan Society of Human Genetics and Springer-Verlag 2006

\begin{abstract}
Cockayne syndrome is a rare autosomal recessive neurodegenerative disorder. It is considered to be a heterogeneous condition based on complementation in cell fusion studies, with two major forms, namely CS-A and CS-B. CKN1 is the gene responsible for CS-A, whose mutations disrupt the transcriptioncoupled repair system of the actively transcribed DNA. Mutation analysis of the CKN1 gene in eight typical CS-A Brazilian patients from six families showed a gene alteration in all of them. We found a total of five novel mutations that were absent from healthy control subjects. Six affected subjects were simple homozygotes and two affected siblings were each compound heterozygotes. While the findings extend the range of mutations in CS-A, there is no obvious genotypephenotype correlation across the mutational spectrum.
\end{abstract}

Keywords Cockayne syndrome type A - CKN1 gene · Neurodegenerative disorders $\cdot$ Mutation analysis ·

Genotype-phenotype correlation

D. R. Bertola · L. M. J. Albano · C. A. Kim

Genetics Clinic Unit, Instituto da Criança do Hospital das

Clínicas, University of São Paulo, Sao Paulo, Brazil

H. Cao · R. A. Hegele

Robarts Research Institute, 406-100 Perth Drive,

London, ON, Canada N6A 5K8

D. P. Oliveira · F. Kok · M. J. Marques-Dias

Department of Neurology, University of Sao Paulo,

Sao Paulo, Brazil

D. R. Bertola $(\varangle)$

Av. Dr. Enéas Carvalho de Aguiar,

647, Sao Paulo 05403-900, Brazil

e-mail: deborarb@icr.hcnet.usp.br

\section{Introduction}

Cockayne syndrome (CS) is a rare autosomal recessive disorder characterized by cachectic dwarfism, premature aging, mental deficiency, microcephaly, intracranial calcifications, neurological degeneration, retinal abnormalities and sensorineural hearing loss. It is considered to be a heterogeneous disorder based on complementation in cell fusion studies: sub-types have been called CS-A and CS-B (Lehmann et al. 1994). The gene that has been shown to be responsible for CS-A-CKN1, also called ERCC8-encodes a Walker domain (WD)-repeat protein (Henning et al. 1995). Mutations in the CKN1 gene disrupt the transcriptioncoupled repair system of the actively transcribed DNA, which is the presumed pathogenic mechanism in CS-A. We analyzed the CKN1 gene in eight typical CS-A patients from six Brazilian families.

\section{Materials and methods}

\section{Subjects}

Eight patients (four males and four females) from six families were evaluated. Two unrelated affected individuals and a pair of affected siblings were each the product of consanguineous mating (families 1, 2 and 3). Informed consent to allow genetic testing was obtained from all participants. The ages of the subjects ranged from 3 years and 8 months to 12 years (mean age of 8 years and 4 months). All patients shared several typical clinical findings (Table 1), including developmental delay, microcephaly, failure to thrive, short stature, enophthalmia, "salt-and-pepper" chorioretinitis, 


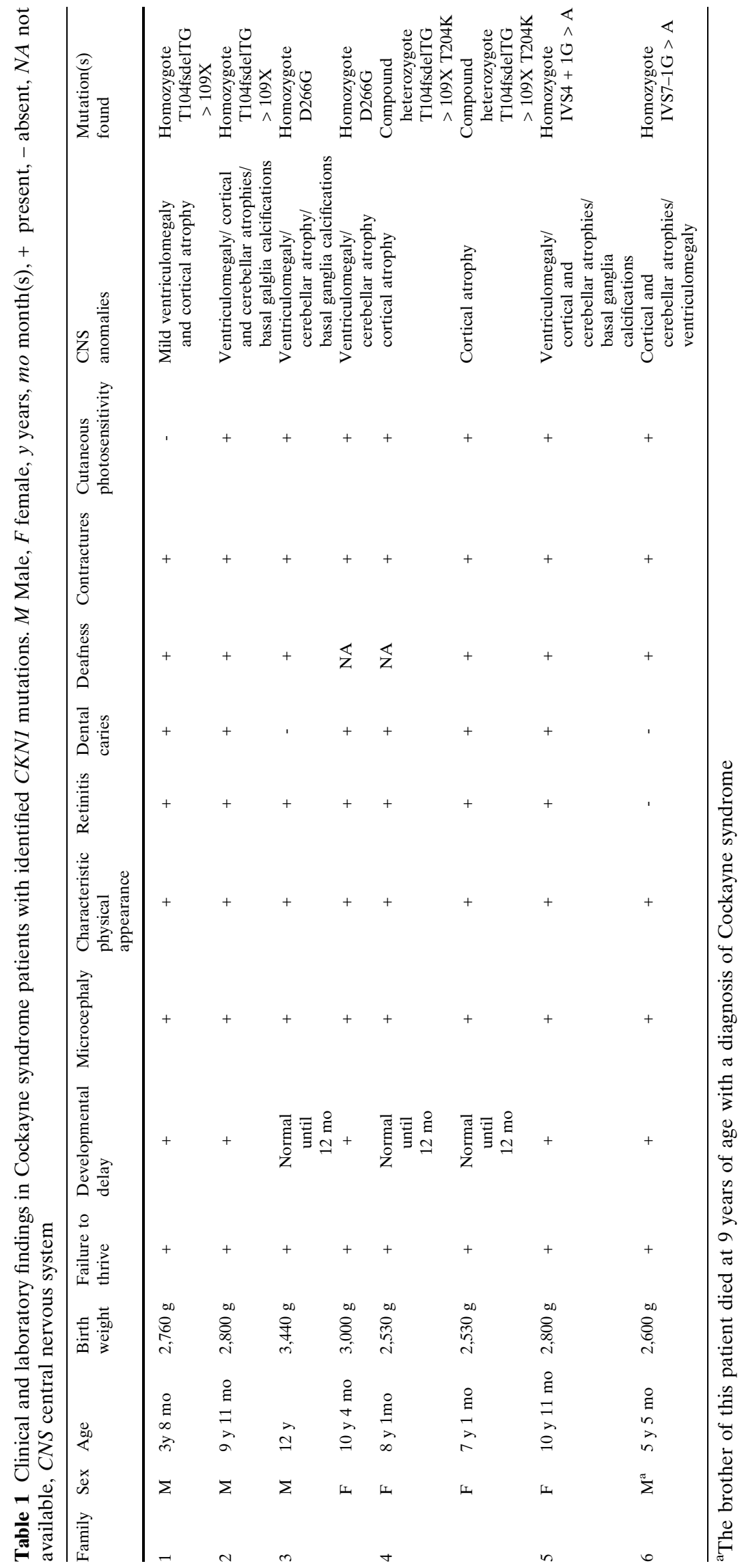


prominent nose, erythematous lesions in the malar and nasal regions, and flexion contractures, especially involving the knees. Neuroimaging studies in affected individuals showed marked cortical atrophy in the majority, ventriculomegaly, atrophy of the cerebellum, and basal ganglia calcifications.

\section{DNA analysis}

In each affected subject, we directly sequenced the 12 exons of the $C K N 1$ gene plus a minimum of 50 nucleotide base pairs (bp) at each intron-exon boundary, which were amplified from genomic DNA using previously reported primers and conditions (Cao et al. 2004). Once a CKN1 mutation was identified in an affected subject, a rapid genotyping method was developed in order to screen healthy control subjects to confirm absence of the mutation from the genomes of 200 healthy control subjects. All genotype reactions were run in parallel with sequence-proven reference standards.

For the IVS4 + 1G > T mutation, we used an allelespecific genotyping method. We amplified a $257 \mathrm{bp}$ fragment containing exon 4 using primers 5 -TCA TCC TAC CTG AGA CTA CTA CTT GTT and 5'- TCC TAC AAA GCA ATC TGC AA. This was followed by treatment with shrimp alkaline phosphatase (SAP) and ExoI to remove primers and unincorporated dNTPs and then followed by ddNTP extension (SnaPShot, PE Applied Biosystems, Mississauga, ON) with primer 5' GGG ATA CAA ATA CAT TAC AA and analysis on a 3730 DNA Sequencer (PE Applied Biosystems, Mississauga, ON).

For the T104fsdelTG > 109X mutation, we amplified a $257 \mathrm{bp}$ fragment containing exon 4 using primers 5'-TCA TCC TAC CTG AGA CTA CTA CTT GTT and 5'- TCC TAC AAA GCA ATC TGC AA. We then digested the amplified fragment with restriction endonuclease $B s r \mathrm{GI}$, and electrophoresed the resulting fragments in $2.5 \%$ agarose gels. Digestion of the wild type allele produced two fragments with sizes 132 and $125 \mathrm{bp}$, while digestion of the mutant allele produced only a single fragment $257 \mathrm{bp}$ in size.

For the T204K mutation, we used an allele-specific genotyping method. We amplified a 294 bp fragment containing exon 7 using primers 5'- TTT GGC CTC ACT TTC TTC AG and 5'- CAA TAC TTC ATT TTA AAG GAG TGA A. This was followed by treatment with SAP and ExoI to remove primers and unincorporated dNTPs and then followed by ddNTP extension (SnaPShot) with primer 5' GAC TAT ATC TTG GCA A and analysis on a 3730 DNA Sequencer.

For the IVS7 $-1 \mathrm{G}>$ A mutation, we amplified a $281 \mathrm{bp}$ fragment containing exon 8 using primers $5^{\prime}$ -
CGA GAC CTC TGT GTG CCA TA and 5'-TGC GTT TAT TAT GTG GCT TCA. We then digested the amplified fragment with restriction enzyme DdeI, and electrophoresed the resulting fragments in $2.5 \%$ agarose gels. Digestion of the wild type allele produced two fragments with sizes 190 and $91 \mathrm{bp}$, while digestion of the mutant allele produced only a single fragment $281 \mathrm{bp}$ in size.

For the D266G mutation, we used an allele-specific genotyping method. We amplified a $256 \mathrm{bp}$ fragment containing exon 9 using primers 5'- CAT CAA ACA TTA AAT ATG TCC TGA A and 5'- AAA TCA AGT GTA TGT CAC AGA TCC A. This was followed by treatment with SAP and ExoI to remove primers and unincorporated dNTPs and then followed by ddNTP extension (SnaPShot) with primer $5^{\prime}$ CTC ACT GTT GGT ACA G and analysis on a 3730 DNA Sequencer.

\section{Results}

DNA analysis

Sequence analysis revealed a total of five novel mutations in our CS-A patients (Table 1; Fig. 1). The TG dinucleotide insertion within CKN1 exon 4 codon 104, which shifted the reading frame and predicted early termination of the protein at 109 amino acids (designated T104fs delTG), was repeatedly found in our cohort: two unrelated cases were homozygous (from families 1 and 2) and both affected siblings from family 4 were compound heterozygotes for this mutation (Fig. 1a). The other mutation in the compound heterozygotes from family 4 was the CKN1 T204K missense mutation. Two affected siblings from family 3 were simple homozygotes for the CKN1 D266G missense mutations. The $C K N 1$ mutation within the splice acceptor site at the exon 3-intron 4 boundary designated IVS4 $+1 \mathrm{G}>\mathrm{A}$, which is predicted to eliminate the splice acceptor recognition sequence, was found in a homozygote from family 5 (Fig. 1b). The CKN1 IVS7-1G > A mutation was found in one homozygous patient from family 6 . We found that all novel CKN1 mutations were absent from the genomes of 200 normal healthy subjects.

\section{Discussion}

To date, fewer than ten reported CS-A patients have been screened for mutations in the CKN1 gene. To the previously reported nonsense mutations E13X and T322X (Henning et al. 1995; Cao et al. 2004; Ridley 

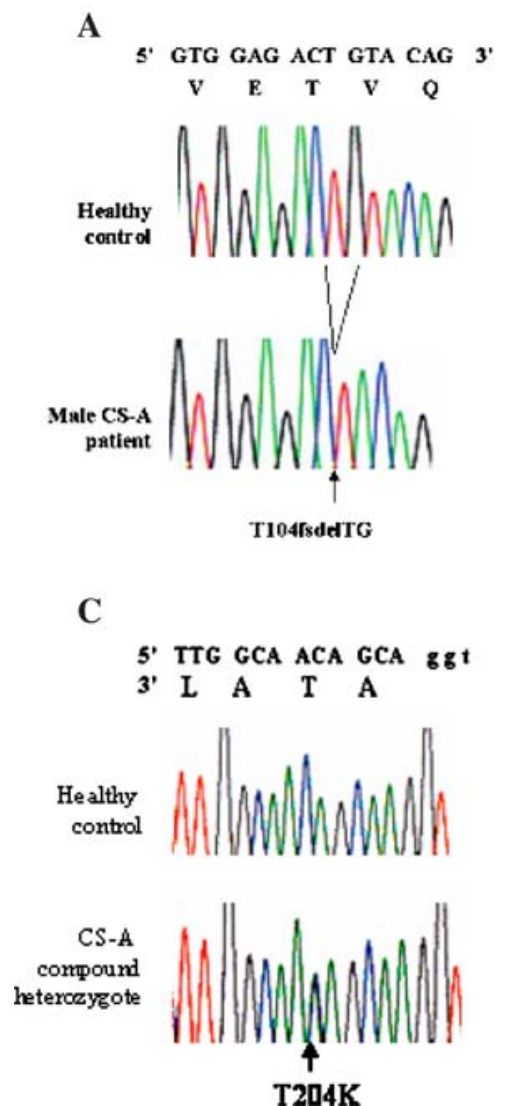

B

5' ACA TTA CAA gtaa 3 .

T L Q
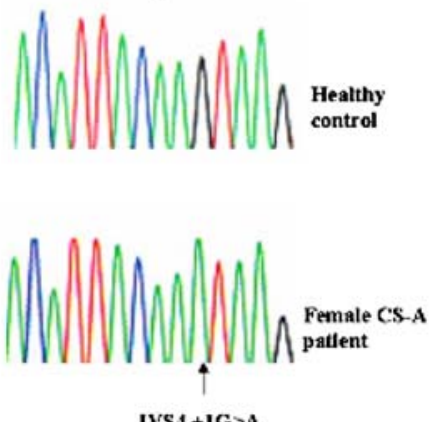

IVS4 +1G $>$ A

D 5't c a g T GCT GAC AGT A 3' A D $\mathbf{S}$

Healthy

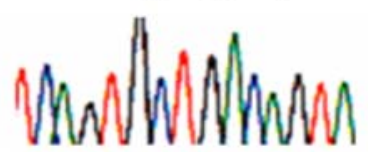

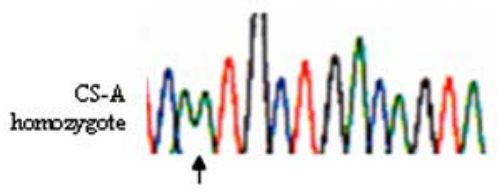

In 7-1 G>A (Homo)
E 5' GGT ACA GAT AAT CGA 3' $\begin{array}{llllll}G & T & \mathbf{D} & \mathbf{N} & \mathbf{R}\end{array}$ Halla

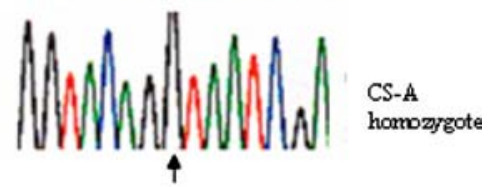

D266G (Homo)
Fig. 1a-e Electropherograms of genomic DNA sequence of the CKN1 mutations. Panels a, b, c, d and e show the T104fsdelTG $>$ 109X (homozygote), IVS4 + 1G > A (homozygote), T204K (heterozygote), IVS7 -G > A (homozygote) and D266G (homozygote) mutations, respectively. The position of the mutant nucleotide sequence is shown with an arrow, and in panel A the deleted nucleotides are indicated. Each panel shows nucleotide sequence (coding in upper case, non-coding in lower case), and single letter codes for amino acid residues. Sequences of the analogous regions from healthy subjects are shown above each mutant sequence for comparison

residue is highly conserved in mammalian species, down to mice, while the D266 residue is conserved in both mammalian and avian species, attesting to the likely functional importance of these two residues. Interestingly, exon 4 has been repeatedly involved as the site for CS-A patient mutations, not just in our cohort but also in other reports (Ren et al. 2003; Komatsu et al. 2004).

There was no obvious difference in phenotype between our subjects with the splicing and frameshift mutations. However, the small total number of molecularly characterized affected CS-A subjects at present precludes an analysis of genotype-phenotype correlation, although our findings expand the spectrum of mutations and mutation types for this disorder.

\section{References}

Cao H, Williams C, Carter M, Hegele RA (2004) CKN1 (MIM 216400): mutations in Cockayne syndrome type A and a new common polymorphism. J Hum Genet 49:61-63 
Henning KA, Li L, Iyer N, McDaniel LD, Reagan MS, Legerski R et al (1995) The Cockayne syndrome group A gene encodes a WD repeat protein that interacts with CSB protein and a subunit of RNA polymerase II TFIIH. Cell 82:555-564

Komatsu A, Suzuki S, Inagaki T, Yamashita K, Hashizume K (2004) A kindred with Cockayne syndrome caused by multiple splicing variants of the CSA gene. Am J Med Genet 128A:67-71

Lehmann AR, Bootsma D, Clarkson SG, Cleaver JE, McAlpine PJ, Tanaka K et al (1994) Nomenclature of human DNA repair genes. Mutat Res 315:41-42
Ren Y, Saijo M, Nakatsu Y, Nakai H, Yamaizumi M, Tanaka K (2003) Three novel mutations responsible for Cockayne syndrome group A. Genes Genet Syst 78:93-102

Ridley AJ, Colley J, Wynford-Thomas D, Jones (2005) Characterisation of novel mutations in Cockayne syndrome type A and xeroderma pigmentosum group $\mathrm{C}$ subjects. J Hum Genet 50:151-154 